\title{
Zinc-Based Fixative Improves Preservation of Genomic DNA and Proteins in Histoprocessing of Human Tissues
}

\author{
Kenneth Wester, Anna Asplund, Helena Bäckvall, Patrick Micke, Andra Derveniece, \\ Ilona Hartmane, Per-Uno Malmström, and Fredrik Pontén
}

Department of Genetics and Pathology (KW, AA, HB, FP), Division of Experimental Urology, Department of Surgical Sciences (KW, P-UM), Uppsala University, and Ludwig Institute for Cancer Research (PM), Uppsala, Sweden; and Clinic of Dermatology and Venerology $(A D, I H)$, Medical Academy of Latvia, Riga, Latvia

SUMMARY: Advantageous preservation of histology and detailed cellular morphology has rendered neutral buffered formalin (NBF) the most widely used fixative in clinical pathology. Despite excellent morphology for routine diagnostics, a major drawback of NBF fixation is its detrimental effect on DNA and RNA quality. In addition to complicating analysis of genes and transcripts in complex tissues, NBF denatures proteins and thereby hampers immunohistochemical visualization of certain antigens. In the present study, we evaluated a zinc-based fixative (ZBF) regarding its effects on tissue morphology, quality of genomic DNA, and preservation of protein immunoreactivity in a broad spectrum of tissues. Four different modes of fixation were analyzed: ZBF-paraffin embedding, NBF-paraffin embedding, ZBF-fixation prior to snap-freezing, and immediate snap-freezing. Laserassisted microdissection, allowing retrieval of a defined number of cells for PCR, was used to study DNA quality. Genomic DNA was analyzed using primers for $\beta 2$-microglobulin and the transferrin receptor. Immunohistochemistry was performed using nine antibodies. Tissue microarray blocks were used for analysis of morphology and immunoreactivity. Only slight impairment of morphologic qualities was found after ZBF-paraffin embedding, whereas ZBF prior to freezing resulted in a more crisp morphology compared with routine cryosections. A significantly higher DNA yield was observed in samples isolated from ZBF-paraffin-embedded tissues compared with NBF-paraffin-embedded tissues. Both yield and quality of DNA was comparable in frozen tissues irrespective to prior ZBF fixation. Immunoreactivity in paraffin-embedded tissue was superior in ZBF-fixated tissue compared with NBF-fixated for a majority of tested antibodies. Furthermore, for seven out of nine antibodies, antigen retrieval pretreatment proved unnecessary in ZBF-fixated tissue. Thus, despite a slight impairment of morphology, ZBF preserves protein structures well. We conclude that ZBF is superior to NBF for analysis of DNA and protein expression. Fixation of tissues in ZBF may also be an alternative strategy to freeze storage of tissue specimens, eg, in future bio-banks. (Lab Invest 2003, 83:889-899).

\begin{abstract}
$\prod$ icroscopic evaluation of a tissue section taken from an excised tumor remains the gold standard for determining a diagnosis of cancer. Analysis of genomic DNA, transcribed genes, and expressed proteins add important information to the histologic features detected in the microscope. To establish optimal treatment and valid prognostic information for individual patients, a synoptic evaluation of morphology in conjunction with analysis of nucleic acids and proteins is important. Evolving knowledge based on the human genome sequence and biochemical pathways, including signaling inside and between cells in a tissue, enable us to determine characteristics of individual tumors.
\end{abstract}

\section{DOI: 10.1097/01.LAB.0000074892.53211.A5}

Received March 28, 2003.

This study was supported by a grant from the Swedish Cancer Foundation. Address reprint requests to: Dr. Kenneth Wester, Rudbeck Laboratory, Uppsala University, SE - 75185 Uppsala, Sweden. E-mail: kenneth.wester@genpat.uu.se
The deleterious effects on DNA and proteins caused by formaldehyde fixation are well known. Initially, there is a reversible and rapid hydroxymethylation of amino and imino groups of the nucleic acid bases, followed by a slow reaction resulting in methylene bridge formation between bases. Formaldehyde also forms cross-linking of proteins to DNA, and the degree of this process depends on the duration of fixation (Crisan and Mattson, 1993; Warford et al, 1988). The variable outcome of PCR and RT-PCR using NBF fixated cells has previously been demonstrated (Williams et al, 1999). Using large numbers of cells as starting material can in part compensate for reduced quality of DNA and mRNA template for PCR. The need for a large number of cells limits the possibility of analyzing defined cell populations due to mixture of cell types in complex tissues. Microdissection of cells, defined under the microscope, from a tissue section ensures a relevant selection of representative cells for subsequent analysis of genomic DNA and mRNA. To retain high quality template, we have earlier used fresh frozen tissues and successfully amplified genomic sequences from a limited number of cells. In these 
studies we were also able to amplify the p53 gene from immunostained sections using only a single cell as template (Ling et al, 2001).

Proteins are altered by cross-linking, mainly in the tertiary and quaternary structure. The changes in immunoreactivity are explained by a reduced availability for antibodies to the secondary structure that is masked by cross-linking of globular proteins (Mason and O'Leary, 1991). The problem of denatured proteins caused by formaldehyde is partly reversible and may be overcome by different strategies of epitope retrieval, eg, enzymatic (Huang et al, 1976) or heatinduced (Shi et al, 1991). For obvious reasons, several alternative fixatives have been suggested (Brenes et al, 1986; Garnis-Jones and Mah, 1988; Goldsworthy et al, 1999; Greer et al, 1991; Herman et al, 1988; Lalani et al, 1995; Shibutani et al, 2000; Weaver et al, 1992). However, none of which has fulfilled the demands according to above. Zinc compounds, as additives to formaldehyde fixatives, are described by several authors (Herman et al, 1988; Tome et al, 1990). Recently, Beckstead (Beckstead, 1994) presented a zinc-based fixative without cross-linking or coagulating agents but with retained morphologic qualities as well as preserved immunoreactivity. These results have also been confirmed by other investigators (Gonzalez et al, 2001; Johansson et al, 2000; Nitta et al, 1997; Wang et al, 1996).

The aim of the present study was to evaluate ZBF as an alternative to NBF preceding paraffin embedding, as well as freeze storage of tissue biopsies. The qualities tested were: (a) preservation of histologic and cytologic detail as observed by light microscopy, (b) preservation of genomic DNA, and (c) preservation of protein structure as measured by immunoreactivity.

\section{Results}

Fixation in ZBF did not introduce any practical inconveniences in pathologic routine laboratory work. In all modes of fixation, tissues were properly dehydrated and also judged as having similar qualities regarding sectioning of the paraffin blocks and frozen tissues.

\section{Morphology}

Formaldehyde fixation is known to reduce the volume of a tissue biopsy by approximately one-third of its true natural volume (Baker, 1958). Using image analysis, we measured the area of each punch biopsy in the array and found that tissues fixated in ZBF prior to embedding in paraffin were smaller in size compared with NBF-fixated parallel biopsies. The difference was approximately the same for all types of tissue and calculated to an average of $37 \%$. Shrinkage was also evident at the cellular level, where nuclei appeared more densely stained and with less detailed chromatin texture (Fig. 1, A and B).

Eight additional fixatives (Table 1) were tested for their effects on morphology, none of which showed substantial benefits compared with ZBF. Therefore, these additional fixatives were not used for further assessment of immunoreactivity or DNA quality.

A more crisp cellular morphology and distinct cohesive histology was found for zinc-based fixation prior to snap-freezing (ZBF-F) tissue compared with immediate snap-freezing (I-F) tissue (Fig. 1, C to D). This was most apparent in biopsies from colon, skin, and liver. Regardless of freezing mode, no difference in morphologic detail or immunoreactivity was observed comparing acetone-fixated and simply air-dried cryosections.

\section{Amplification of Genomic DNA}

Paraffin-embedded tissue fixated in ZBF resulted in DNA yields considerably higher compared with NBFfixated tissue (Fig. 2). Amplification of $\beta 2-$ microglubulin and transferrin receptor gene fragment show that frozen tissue (ZBF-F and I-F), yield the greatest concentrations of DNA (Fig. 2). Only incremental amounts of PCR fragments compared with negative controls were observed for tissues fixated in NBF. Regardless of fixation mode, no difference was found between different fixation times ( 1 and 10 days).

\section{Size Distribution and Quantification of Total RNA}

We investigated if different fixation methods induced fragmentation or degradation of RNA. The length distribution and quantification of RNA molecules were determined with a bioanalyzer (Agilent Technologies, Palo Alto, California). The RNA isolated from I-F and ZBF-F tissue displayed a normal size spectrum, including clearly visible $18 \mathrm{~S} / 28 \mathrm{~S}$ rRNA peaks (Fig. 3, A and $B$ ), indicative for a limited degradation or fragmentation of RNA. Electropherograms of the samples, which were paraffin-embedded (ZBF-1; ZBF-10, NBF-1, and NBF-10), showed a pattern of pronounced degradation of RNA transcripts to fragments smaller than 200 bp in size (Fig. 3, C to F). The comparison of RNA content calculated by the Bioanalyzer software revealed the same pattern of degradation (Table 2). Frozen tissue samples (I-F, ZBF-F) contained the highest amount of RNA, whereas only limited amounts were detected in the paraffin-embedded tissues (ZBF-1; ZBF-10, NBF-1, and NBF-10). Thus, the Bioanalyzer analysis indicated that histoprocessing and/or storage in paraffin is responsible for the degradation of RNA. In contrast, the zinc-based fixation itself does not appear to affect RNA integrity.

\section{Immunohistochemistry}

For paraffin-embedded tissues, ZBF proved superior to NBF (Fig. 4, A to D) in preservation of epitopes targeted by antibodies recognizing epithelialcadherin, CD 31, EGFR, HER-2, and cytokeratin; whereas the immunoreactivity for $\mathrm{Ki}-67$ and p53 protein was similar regardless of fixation mode. For immunostaining of cyclin B1 and p27 $7^{\mathrm{KIP} 1}$, NBF-fixated tissue showed higher immunoreactivity compared with ZBF-fixated tissue (Fig. 4, E and F). For eight antibodies in the panel, NBF-fixated tissue required 

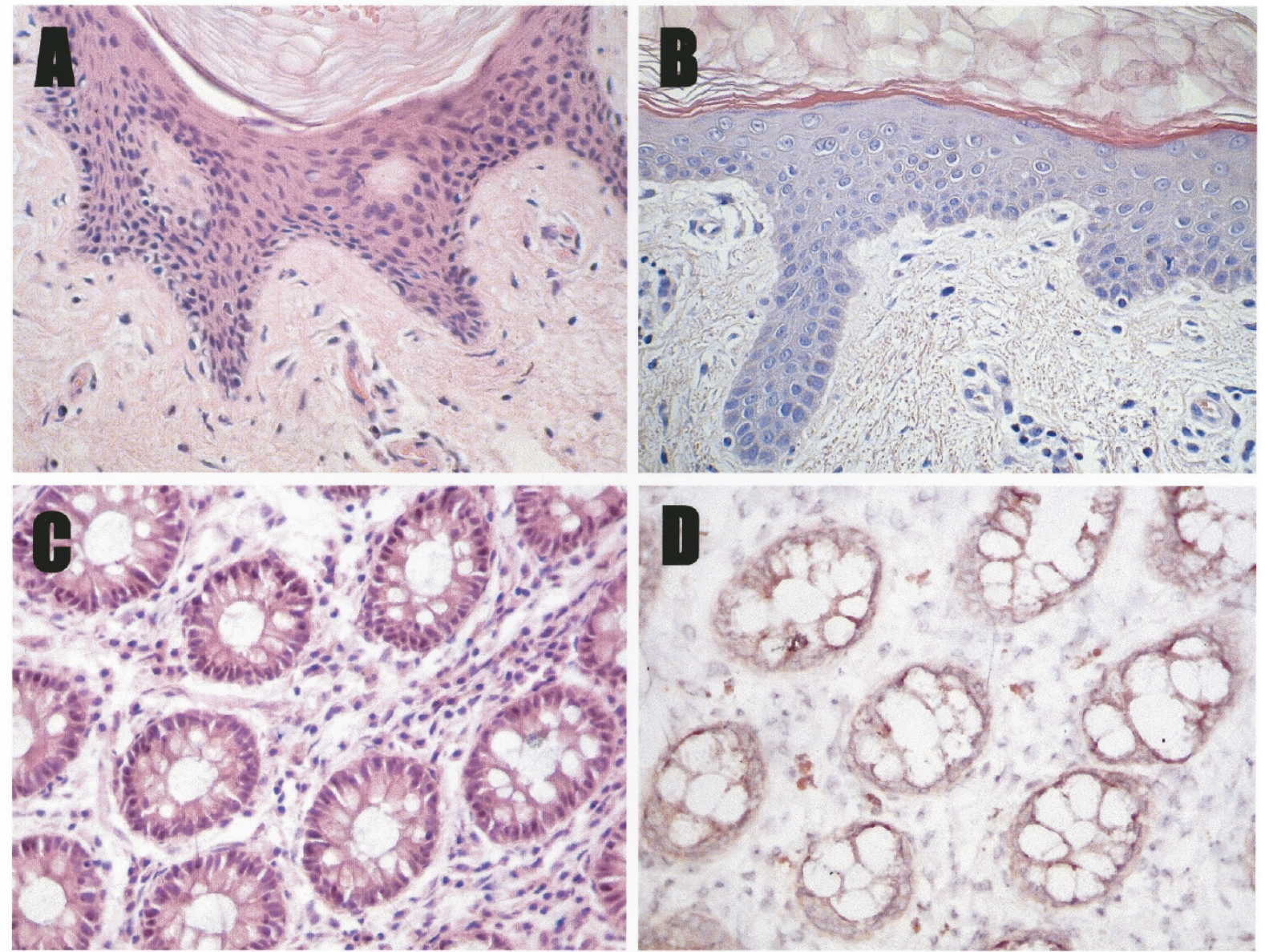

\section{Figure 1}

Hematoxylin-eosin staining of paraffin sections from a skin biopsy, illustrating the shrinkage of cells in tissues fixated in ZBF (A) compared with NBF (B) Hematoxylin-eosin-stained cryosections from a colon biopsy soaked in ZBF prior to freezing (C) and immediately frozen (D). Observe the crisp and cohesive morphology in tissue soaked in ZBF (C) compared with tissue frozen immediately (D).

\section{Table 1. Alternative and Modified Zinc-Based Fixatives Were Tested in a Limited Number of Tissues for Comparison with ZBF and NBF}

95\% ethanol + Polyethylene glycol, Mol Wt 200 (1:1) NeoFix (Merck, Darmstadt, Germany)

ZBF in $25 \mathrm{~mm}$ Tris

ZBF in $50 \mathrm{~mm}$ Tris

ZBF and followed by incubation in $30 \%$ sucrose for $2 \mathrm{hr}$ ZBF containing 10\% Polyethylene glycol, Mol Wt 200

ZBF containing $0.06 \%$ spermine tetrahydrochloride

ZBF containing $10 \mathrm{~mm}$ EDTA

ZBF prepared as described in "Material and Methods" NBF prepared as described in "Material and Methods"

ZBF, zinc-based fixative; NBF, neutral buffered formalin.

Where not otherwise stated, chemicals were purchased from Sigma, St. Louis, Missouri. For all fixatives, tissues were fixated for 24 hours at room temperature before dehydration and paraffin embedding.

heat-induced or enzymatic epitope retrieval to restore immunoreactivity (Table 3). For six of these eight antibodies, immunoreactivity was preserved without epitope retrieval in ZBF-fixated tissues (Fig. 5). The results are summarized in Table 4.
Cryosections were immunostained using a limited number of antibodies, epithelial-cadherin, CD 31, EGFR, and pan-cytokeratin. An only minor difference in immunoreactivity was observed between the two modes of cryopreservation.

\section{Discussion}

An optimal fixative should provide an excellent histologic and morphologic detail for routine light microscopical examination and preservation of nucleic acids and proteins. Fixation of tissue is important to obtain the high quality of morphology needed for accurate histopathologic diagnosis. Diseased and normal tissue represents the disease history of a patient and thus constitutes a valuable source of information for both retrospective and prospective clinical studies. Assessment of prognostic markers and evaluation of tumor response to new therapeutic drugs are two examples in which clinical biopsy material is essential. The most widely used fixative is NBF, which unfortunately can disguise nucleic acids and proteins thus disabling analysis of genes and gene function. Therefore, there is an urgent need for better preservation of cellular components in modern 

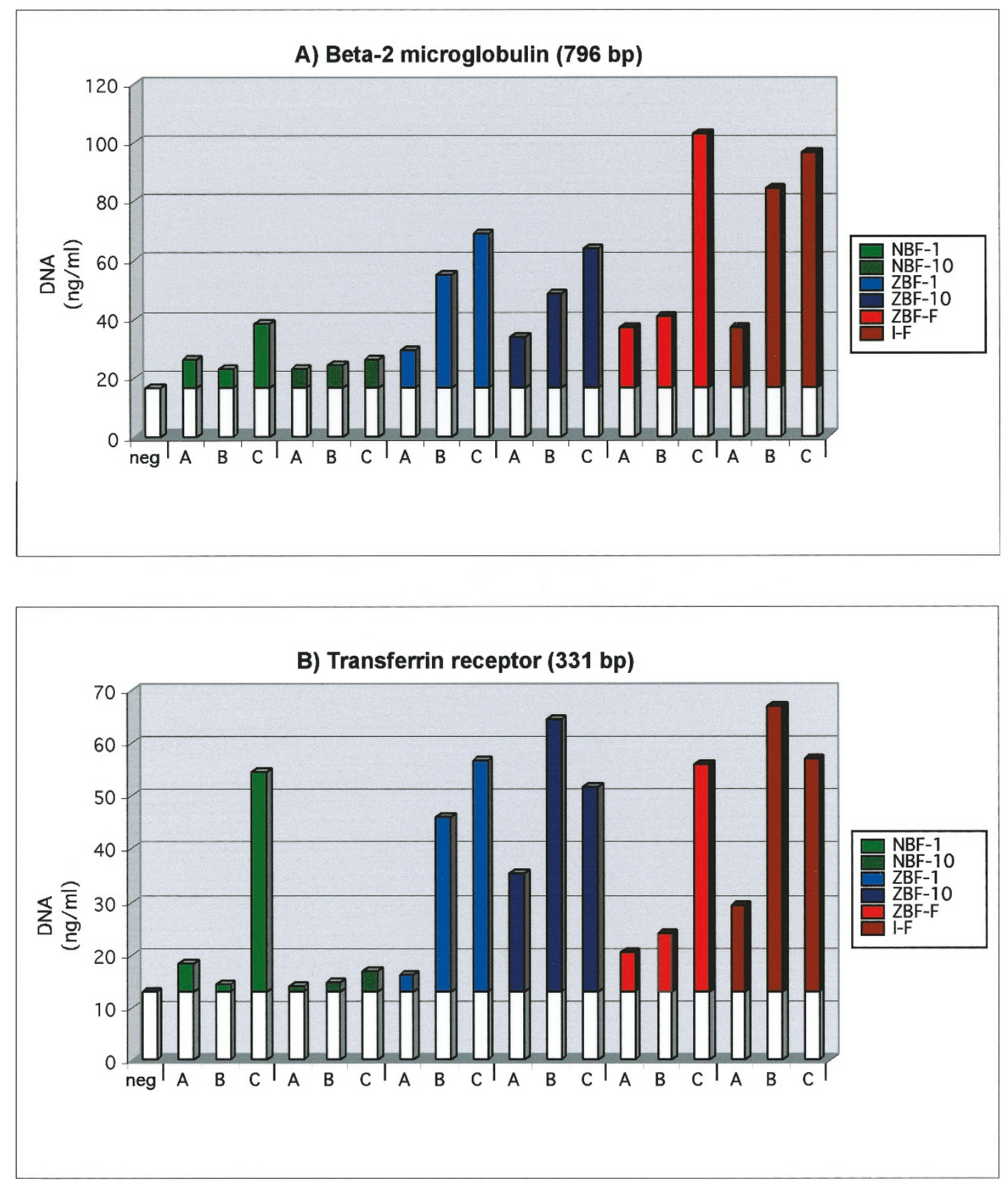

Figure 2.

Results from measurements of DNA concentration after amplification of $\beta$-2 microglobulin (A) and transferrin receptor (B) gene fragments from microdissected cells retrieved from various fixated tissues. $F$ denotes frozen tissue; 1 denotes 1 day (24 hours) and 10 denotes 10 days (240 hours). Each bar represents the mean of four fluorimetric measurements of Picogreen incorporation into dsDNA (two PCR reactions, each measured twice). The negative control comprises PCR reactions without addition of DNA. This was included to enable an estimation of background, caused primarily by primer-dimers. $A, B$, and $C$ denote the volume of microdissected cell populations, $1.1 \times 10^{5} \mu \mathrm{m}^{3}, 3.0 \times 10^{5} \mu \mathrm{m}^{3}$, and $4.4 \times 10^{5} \mu \mathrm{m}^{3}$, respectively. Note outlier NBF-1 in Figure $2 \mathrm{~B}$ showing a remarkable high yield, consistent with unpredictable quality of NBF-fixated tissue.

clinical pathology, using molecular biology, in situ hybridization, and immunohistochemistry.

The use of ZBF has proven advantageous in immunohistochemical staining of paraffin-embedded tissue sections (Beckstead, 1994; Gonzalez et al, 2001; Nitta et al, 1997; Wang et al, 1996). Furthermore, according to Beckstead (Beckstead, 1994) no apparent loss of antigen was observed in paraffin blocks stored for over 3 years at room temperature. These studies have focused on lymphoid tissues and epitopes on immune system cells. ZBF has also been used for evaluation of transcription profiles from microdissected cell populations (Scheidl et al, 2002). In this recently published study, ZBF was found to efficiently recover and preserve the integrity of RNA. According to our experience, using ZBF does not introduce any practical 

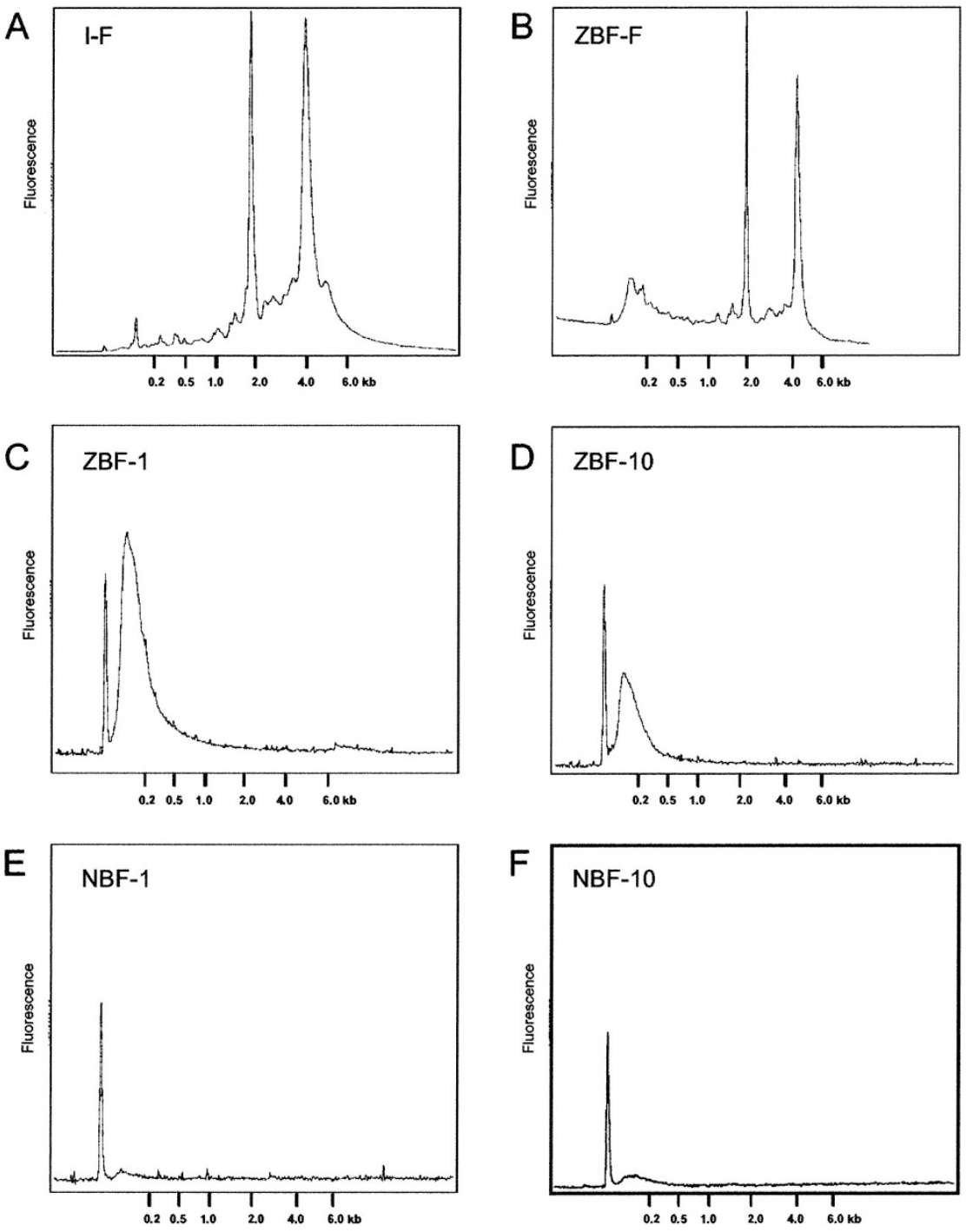

Figure 3.

Representative electropherograms of total RNA from tissue immediately frozen (I-F) (A), tissue fixated in zinc-based fixation prior to snap-freezing (ZBF-F) (B), ZBF-1 (C); ZBF-10(D), neutral buffered formalin (NBF)-1(E), NBF-10 (F). The RNA was extracted from abraded tissue sections and separated with an RNA 6000 pico Labchip in a Agilent 2100 Bioanalyzer. The size range of RNA transcripts is estimated by the overlay function in the bioanalyzer software. Migration times of the ladder RNA are indicated of the bottom of each graph.

inconveniences in pathologic routine laboratory work. On the contrary, ZBF is nonhazardous, easy to prepare, inexpensive, and requires no special arrangements for storage.

Results from PCR amplifications of both $\beta 2$ microglobulin and transferrin receptor gene fragments show that fixation in ZBF, prior to either freezing or embedding in paraffin, results in good preservation of genomic DNA. Consistently higher DNA concentrations are found with increasing number of cells following ZBF fixation and I-F compared with NBF fixation (Fig. 2). Our results suggest that the poor amplification of DNA fragments extracted from NBF-fixated tissues are a result of poor DNA quality. This hypothesis is supported by the observation that, for NBF samples contrary to ZBF samples, no obvious increase in amplification end-product was seen with an increased starting volume of tissue.
Table 2. Comparison of RNA Content

\begin{tabular}{lc}
\hline $\begin{array}{c}\text { Fixation } \\
\text { procedure }\end{array}$ & Mean $\pm \mathrm{sD}$ \\
\hline I-F & $1750 \pm 1200 \mathrm{ng}$ \\
ZBF-F & $1765 \pm 1433 \mathrm{ng}$ \\
ZBF-1 & $256.75 \pm 299 \mathrm{ng}$ \\
ZBF-10 & $13.5 \pm 7 \mathrm{ng}$ \\
NBF-1 & $12.25 \pm 12 \mathrm{ng}$ \\
NBF-10 & $12.25 \pm 12 \mathrm{ng}$ \\
\hline
\end{tabular}

Snap-frozen tissue sections (I-F) and sections treated with five different modes of fixation (ZBF-F, ZBF-1, ZBF-10, NBF-1, NBF-10) were abraded from glass slides and dissolved in lysis buffer for RNA extraction. RNA was separated and quantified with an RNA 6000 Pico Labchip in a Bioanalyzer. Results were obtained from four independent experiments.

Laser-assisted microdissection allowed defined areas of cell populations to be collected (Fig. 6). Con- 

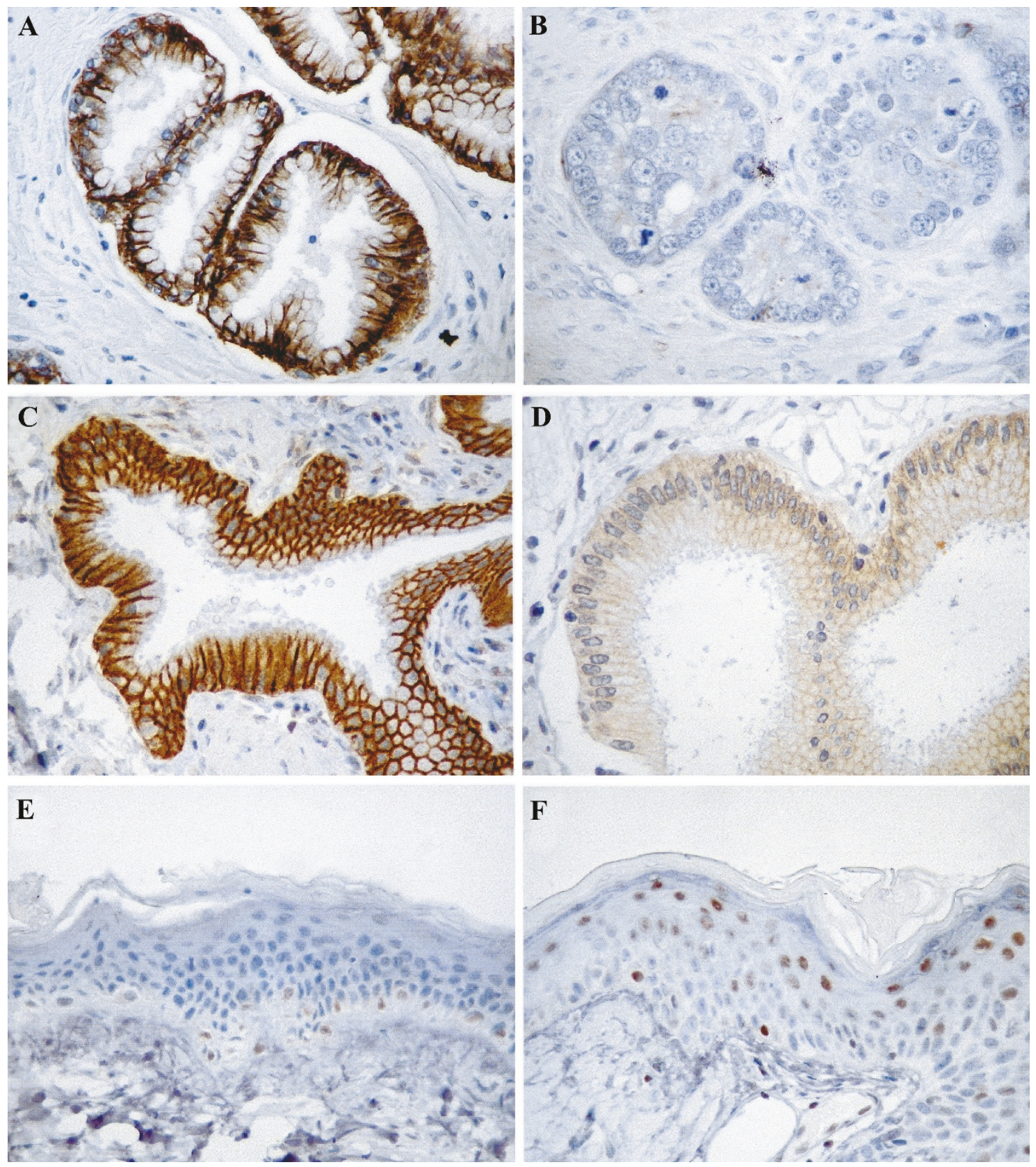

\section{Figure 4.}

Immunostaining for epithelial cadherin in glandular epithelium of the prostate, fixated in zinc-based fixative (ZBF) (A) and neutral buffered formalin (NBF) (B) Analogous, images $C$ (ZBF) and D (NBF) show gall bladder epithelium stained for HER-2 receptor. Note the intense staining in tissue fixated in ZBF compared with NBF. However, p27 ${ }^{\text {KIP27 }}$ immunostaining of a skin biopsy fixated in ZBF (E) showed only weak staining, whereas tissue fixated in NBF (F) resulted in an intense staining of mainly superficial cells of the dermis. All images (A to $F$ ) represent paraffin sections.

sequently, due to mode of fixation, variability in cell number among the samples was found. Shrinkage was more pronounced in tissues fixated in ZBF compared with NBF, and thus, the number of microdissected cells was higher in ZBF samples than in NBF samples. The relative cell density in microdissected samples was 3:2 for both ZBF:NBF (paraffinembedded tissue) and ZBF-F:I-F (frozen tissue). Although this inconsistency in the amount of starting material must be taken into consideration, it does not fully explain the higher amplification efficacy in ZBF samples compared with NBF samples.

In the present study, we noticed an unexpected and unaccountable result as seen in Figure 2B, where one of the bars representing DNA yield from amplification of NBF-fixated tissue is remarkably high. It is well known that NBF fixation leads to incoherent PCR results when only a low amount of template is available for amplification of DNA. The use of NBF-fixated tissue for DNA amplification in such cases is a predic- 
Table 3. Characteristics of Antibodies and Their Corresponding Antigen/Epitope

\begin{tabular}{|c|c|c|c|c|c|}
\hline Antibody & Clone $^{a}$ & $\begin{array}{l}\text { Target protein } \\
\text { Mw (kDa) }\end{array}$ & Dilution & Epitope retrieval $^{b}$ & Source \\
\hline Ki 67 & MIB1 & 345,395 & $1: 300$ & $+/-$ HIER & $\begin{array}{l}\text { Immunotech, Marseille, } \\
\text { France }\end{array}$ \\
\hline Cyclin B1 & 18 & 60 & $1: 1000$ & $+/-$ HIER & $\begin{array}{l}\text { Transduction Lab., Lexington, } \\
\text { Kentucky }\end{array}$ \\
\hline p53 & D07 & 53 & $1: 200$ & $+/-$ HIER & DAKO \\
\hline p27 & K25020 & 27 & $1: 1000$ & $+/-$ HIER & Transduction Lab. \\
\hline Epithelial-cadherin & 36 & 120 & $1: 1.000$ & - HIER & Transduction Lab. \\
\hline CD31 & JC70 & $120-130$ & $1: 80$ & $+/-$ HIER & DAKO \\
\hline EGFR & $31 \mathrm{G} 7$ & 170 & $1: 40$ & +/- Enzymatic retrieval & $\begin{array}{l}\text { Zymed, South San Francisco, } \\
\text { California }\end{array}$ \\
\hline HER-2 & Rabbit polyclonal & 185 & $1: 300$ & $+/-$ HIER & DAKO \\
\hline Pan-cytokeratin & AE1/AE3 & $40-68$ & $1: 1.000$ & $+/-$ HIER & $\begin{array}{l}\text { Roche Molecular } \\
\text { Biochemicals, Mannheim, } \\
\text { Germany }\end{array}$ \\
\hline
\end{tabular}

HIER, heat-induced epitope retrieval.

${ }^{a}$ Mouse monoclonal antibodies where not otherwise stated.

${ }^{b}$ Based on available immunostaining protocols for NBF-fixated tissue, two alternative modes of epitope retrieval were used. HIER in citrate buffer or enzymatic epitope retrieval by protease treatment. These protocols are described in detail in the "Material and Methods" section.

ament, and problems including inconsistency and error rates have been described previously (Williams et al, 1999).

Depending on fixation time and length of amplicon, NBF disables successful amplification of DNA. This is most likely the result of DNA fragmentation, as shorter fragments are successfully amplified to a greater extent than longer fragments, particularly in tissues fixated for a long time in NBF (Greer et al, 1994). This is in concordance with our results, where the amplification efficacy for the 796 bp $\beta 2$-microglobulin fragment in NBF-fixated material is close to base-line levels. This is further supported by the more variable results seen for the shorter transferrin receptor gene fragment. We must also bear in mind, however, that possible loss of material during microdissection and the random nature of "pick-up" efficacy primarily during the first cycles of PCR affect the outcome of each reaction (Persson et al, 2000).

To test the effect on RNA preservation and integrity of the different fixatives, cells from thin tissue sections were scraped off glass slides and processed. We found no differences comparing IF and ZBF-F, suggesting that immersion of tissue in ZBF for 2 hours does not impair analysis of RNA. Although a lower amount of RNA could be extracted from ZBF-1 (Table 2), our data show a clear degradation pattern for all paraffin-embedded samples. RNA extractions were also tested using real-time PCR (GADPH), with similar results as shown for microchip gel electrophoresis. In addition, experiments using microdissected samples (1000 cells) and microchip gel electrophoresis as well as real-time PCR resulted in too little yield of RNA to allow any firm conclusions (data not shown).

Different chemical fixatives have different modes of action, ie, coagulative and cross-linking. However, most fixatives have in common that they are originally designed for an optimal histologic exami- nation. For immunorecognition there is no best fixative of all antigens; rather an antigen and antibody clone and a fixative-processing method must be matched to optimize immunorecognition (Arnold et al, 1996). This fact probably explains why some proteins in this study are better preserved in ZBF, whereas others are better preserved in NBF. Small proteins such as $\mathrm{p} 27^{\mathrm{KIP} 1}$ may easily diffuse from the tissue and therefore require extensive cross-linking fixation such as NBF. On the contrary, certain epitopes located within larger proteins (eg, epithelial-cadherin and CD31) may be masked by NBF and thus preferably require coagulative fixation, which to a lesser degree distorts the secondary structure of proteins. Interestingly, this hypothesis is partly confirmed by our results. As seen in Table 4, most proteins best preserved in ZBF are of high molecular weight, whereas proteins best preserved in NBF are of lower molecular weight.

We conclude that ZBF preserves high yield and high quality of genomic DNA without negative effects on RNA and with only slight impairment of morphology. In addition, it allows for high quality immunohistochemistry, and for some antibodies, abrogates the need for antigen retrieval pretreatment. Furthermore, the ZBF offers a practical strategy for an organized collection and storage of tissue, eg, in bio-banks. This is customarily done by freeze storage and subsequent cryosectioning and thus, requires low-temperature freezers and cryostates. The presented alternative using ZBF not only eliminates the need for expensive equipment but also improves both morphologic and histologic details in the tissue. The described method should prove a useful strategy when analyzing the same tissue specimen for morphology, protein expression, genomic DNA, and RNA. 


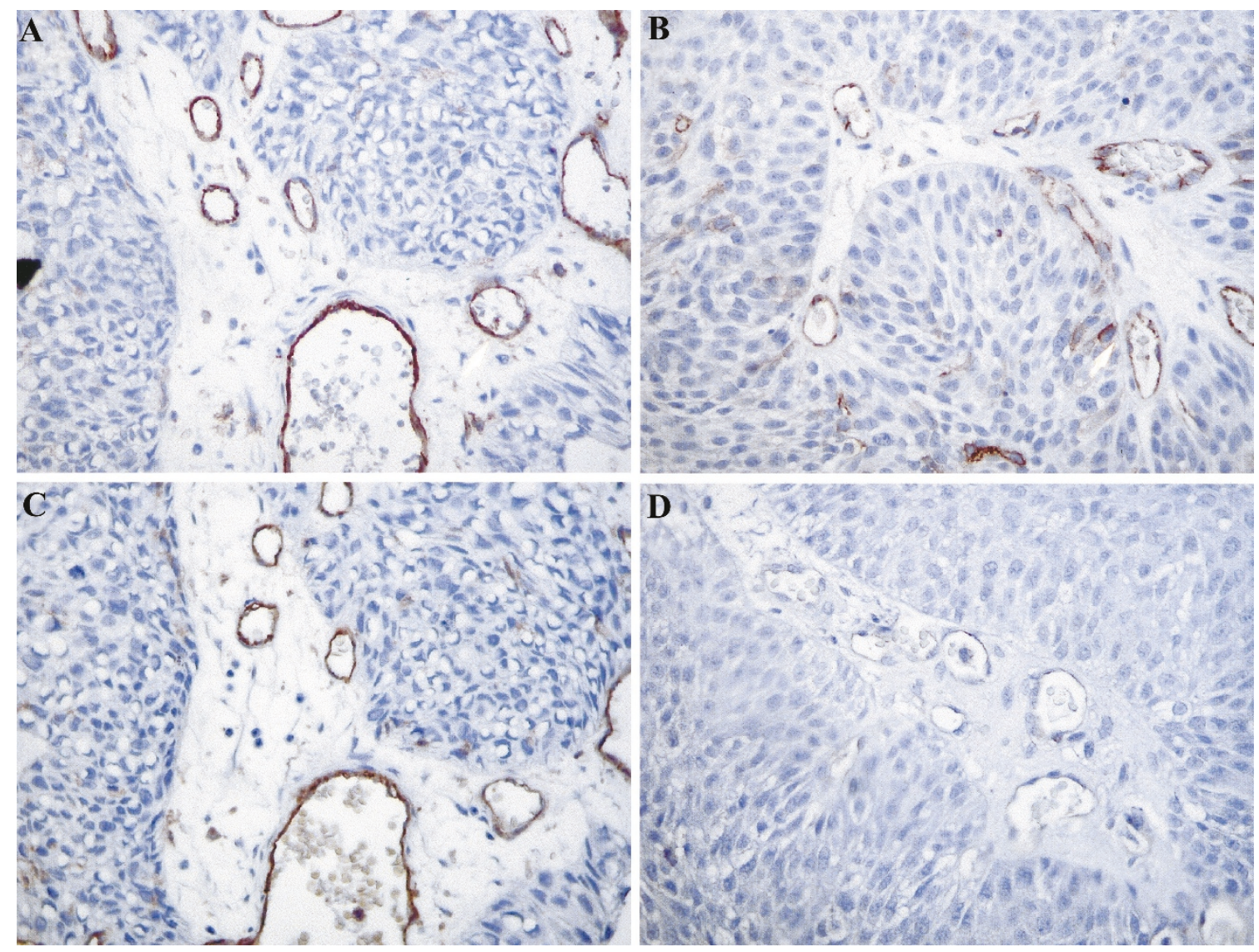

Figure 5.

Transitional cell carcinoma of the bladder immunostained for endothelial cells using antibody against CD31. Stainings were done with heat-induced epitope retrieval ( $A$ and $B$ ) and also without retrieval $(C$ and $D)$. Sections from tissue fixated in zinc-based fixative ( $A$ and $C$ ) show an equally high expression of CD31 in both modes of staining, whereas immunoreactivity in sections from tissue fixated in neutral buffered formalin (B and $D)$ is reduced when retrieval is excluded (D). All images (A to $\mathrm{D})$ represent paraffin sections.
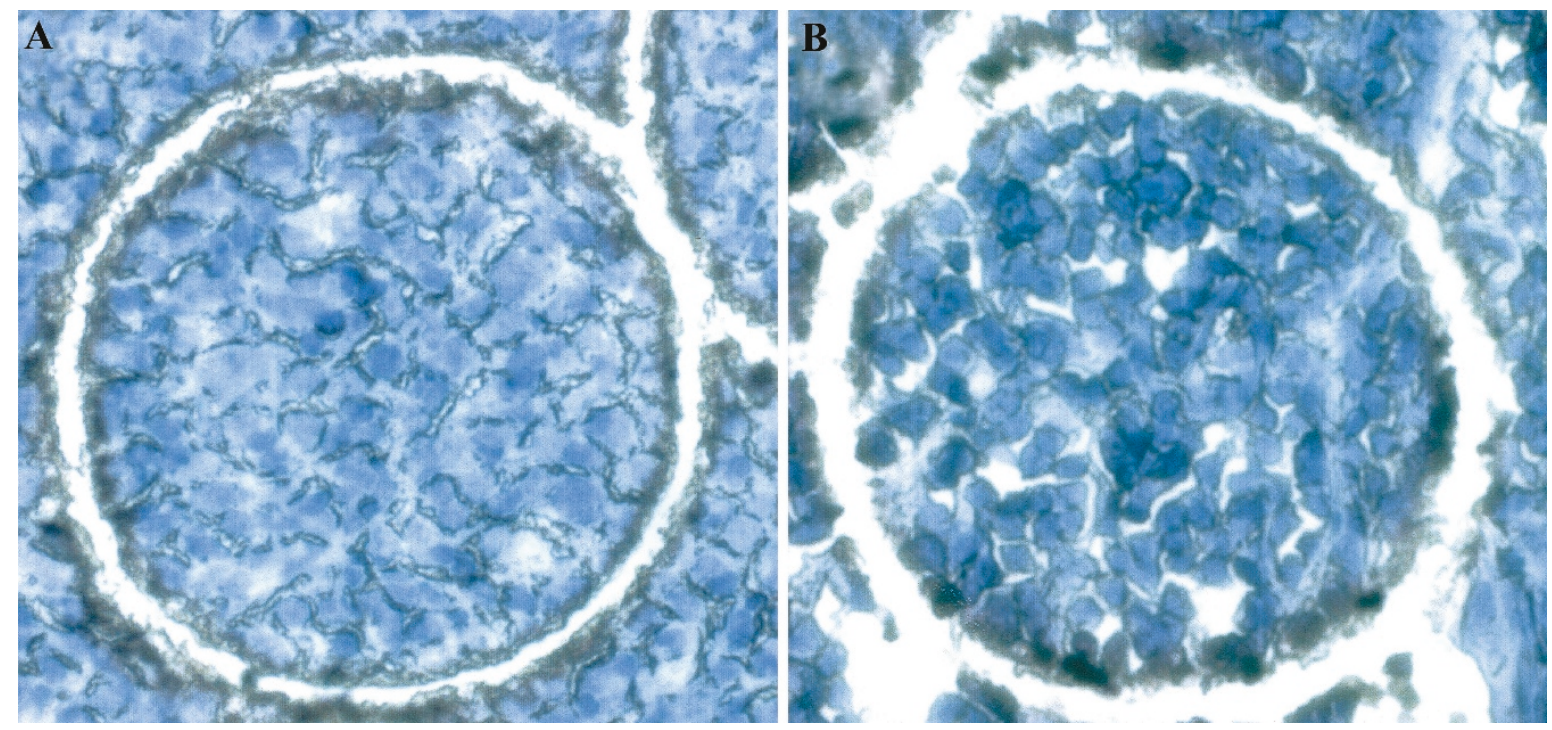

Figure 6.

Laser-assisted microdissection of lymphocytes from a hematoxylin-stained cryosection of normal tonsil, immediately frozen (A) and soaked in zinc-based fixative prior to freezing (B). The circles are $110 \mu \mathrm{m}$ in diameter $\left(1.1 \times 10^{5} \mu \mathrm{m}^{3}\right)$ and represent approximately 120 cells $(\mathrm{A})$ and, due to shrinkage, 180 cells $(B)$. 
Table 4. The Semi-Quantitatively Judged Differences in Immunoreactivity of Paraffin-Embedded Tissues Fixated in ZBF or NBF ${ }^{a}$

\begin{tabular}{lcc}
\hline & $\begin{array}{c}\text { Comparison of } \\
\text { immunoreactivity }\end{array}$ & $\begin{array}{c}\text { Evaluated } \\
\text { Antibodies }\end{array}$ \\
\cline { 2 - 3 } tissues $(n)^{d}$
\end{tabular}

\section{Materials and Methods}

\section{Tissue Sampling and Processing}

Fresh human tissues from various organs (Table 5) were obtained from surgery at the University Hospital in Uppsala, Sweden. At arrival to the Department of Pathology, six approximately 3-mm thick pieces were cut from each tissue, out of which four were placed in cassettes for further processing according to the following schedule: (a) ZBF-1: fixation for 24 hours at room temperature in ZBF, containing $0.5 \%$ zinc chloride, $0.5 \%$ zinc acetate in $0.1 \mathrm{M}$ Tris base buffer containing $0.05 \%$ calcium acetate, $\mathrm{pH} 7.4$; (b) ZBF-10: fixation for 10 days at room temperature in ZBF; (c) NBF-1: fixation for 24 hours at room temperature in $10 \%$ NBF; and (d) NBF-10: fixation for 10 days at room temperature in $10 \% \mathrm{NBF}$.

After fixation, all tissue specimens were processed according to standard procedures and were paraffin embedded. These paraffin blocks served as donor blocks for the tissue array construction. The remaining two pieces were prepared for snap-freezing and subsequent storage at $-70^{\circ} \mathrm{C}$ according to the following schedule: (a) ZBF-F: after incubation in ZBF for 2 hours at room temperature, the tissue was embedded in OCT (Miles Inc., Elkhart, Indiana) in a plastic cryomold (Miles Inc.) and was snap-frozen (F); and (b) I-F: The tissue was immediately (I) placed in a plastic embedding cryomold, covered with OCT and snap-frozen.

\section{Tissue Array Construction}

Biopsies from 14 normal and 5 tumor tissues (Table 5) were represented in the donor blocks. Hematoxylin-
Table 5. Tissue Sampling Results of Various Organs

\begin{tabular}{llr}
\hline \multicolumn{1}{c}{ Tissue } & Normal (N)/Tumor $(\mathrm{T})$ & $\mathrm{N}$ \\
\hline Tonsil & $\mathrm{N}$ & 3 \\
Skin & $\mathrm{N}$ & 2 \\
Skin & $\mathrm{T}$ & 1 \\
Liver & $\mathrm{N}$ & 1 \\
Gall bladder & $\mathrm{N}$ & 1 \\
Colon & $\mathrm{N}$ & 1 \\
Colon & $\mathrm{T}$ & 1 \\
Kidney & $\mathrm{N}$ & 1 \\
Stomach & $\mathrm{N}$ & 1 \\
Esophagus & $\mathrm{N}$ & 1 \\
Prostate & $\mathrm{N}$ & 1 \\
Lung & $\mathrm{N}$ & 1 \\
Lung & $\mathrm{T}$ & 1 \\
Thyroid gland & $\mathrm{N}$ & 1 \\
Lymphoma & $\mathrm{T}$ & 1 \\
Urinary bladder & $\mathrm{T}$ & 1 \\
Total & $\mathrm{N}=14, \mathrm{~T}=5$ & 19 \\
\hline
\end{tabular}

eosin and Weigert van Gieson-stained sections from each donor block were used to select appropriate areas for the tissue array construct. Using a tissue arrayer (Beecher Instruments, Silver Spring, Maryland), three ø 1.0-mm punches were taken from each donor block. In all, a total number of 228 punches (19 tissue samples $\times 4$ modes of fixation $\times 3$ punches) were taken and distributed into three final tissue array recipient blocks.

\section{Additional Fixatives}

In a limited number of tissues, an additional eight fixatives were tested for their effect on morphology and for comparison, ZBF-1 and NBF-1 were also included. This was done on separately histoprocessed tissues, fixated for 24 hours. The compositions of the fixatives are described in detail in Table 1.

\section{Cryosectioning}

Cryosections for morphologic examination and immunohistochemistry were cut at $4-\mu \mathrm{m}$ thickness and placed onto Superfrost plus slides (Mentzel, Braunschweig, Germany). Sections were air-dried for 1 hour or air-dried and fixated in acetone for 20 minutes followed by a further 1-hour air-dry before storage at $-20^{\circ} \mathrm{C}$. Histologic stainings and immunohistochemistry were done according to the protocols for paraffinembedded tissue sections.

Cryosections for subsequent microdissection were cut at $12-\mu \mathrm{m}$ thickness and placed onto uncoated standard slides, briefly air-dried and stored at $-20^{\circ} \mathrm{C}$. Immediately prior to microdissection, sections were thawed and stained in hematoxylin to enable histologic orientation.

\section{Microdissection}

Microdissection was performed on $12-\mu \mathrm{m}$ sections from tonsil using the PALM Robot-Microbeam laser 
microdissection system (P.A.L.M. GmbH, Bernried, Germany). The system, based on a sharp-focused laser beam, allows high precision dissection of defined areas of cells from a tissue section (Schutze and Clement-Sengewald, 1994). From each 12- $\mu \mathrm{m}$ section, three samples of different volumes were prepared: $1.1 \times 10^{5} \mu \mathrm{m}^{3}, 3.0 \times 10^{5} \mu \mathrm{m}^{3}$, and $4.4 \times 10^{5}$ $\mu \mathrm{m}^{3}$ (Fig. 6). Dissected material was subsequently removed from the slide using a small scalpel and placed in $30 \mu \mathrm{l}$ of $1 \times \mathrm{PCR}$ buffer and $1 \mathrm{mg} / \mathrm{ml}$ of proteinase $\mathrm{K}$. To check reproducibility, an additional sample of $4.4 \times 10^{5} \mu^{3}$ was microdissected and analyzed from the freezing modes ZBF-F and I-F. The results from these repeated microdissections were almost identical.

\section{Amplification}

To investigate the amount and quality of amplifiable DNA, we performed PCR reactions for two genes, the transferrin receptor (331 bp product) and $\beta 2-$ microglubulin (796 bp product). Each sample was analyzed for both genes, and two reactions were run for each gene. The reactions for both the transferrin receptor and $\beta 2$-microglubulin contained $6 \mu$ l of DNA, $1 \times$ PCR buffer, $1.75 \mathrm{~mm} \mathrm{Mg}^{2+}, 0.2 \mathrm{~mm}$ of dNTP, 4 pmols of each primer, and $1 \mathrm{U}$ of Taq Gold (Applied Biosystems, Foster City, California). The program used was as follows: initial activation of Taq Gold at $95^{\circ} \mathrm{C}$ for 10 minutes; 10 cycles of $95^{\circ} \mathrm{C}$ for 1 minute, annealing temperature for 5 minutes and $72^{\circ} \mathrm{C}$ for 3 minutes; 25 cycles of $95^{\circ} \mathrm{C}$ for 1 minute, annealing temperature for 1.5 minutes, and $72^{\circ} \mathrm{C}$ for 2 minutes; and final extension at $72^{\circ} \mathrm{C}$ for 10 minutes. The annealing temperature for $\beta 2$-microglobulin was $55^{\circ} \mathrm{C}$, and for the transferrin receptor $59^{\circ} \mathrm{C}$. The primer sequences for $\beta 2-$ microglubulin was MG-F $5^{\prime}-$ GTGGAGCATTCAGACTTGTC, MG-R 5'- CTCCATGATGCTGCTTACATG; and for the transferrin receptor TFR-F 5'- GAAGCATCCGGTTACTGGG och TFR-R 5'AGAAACTGCTGGGATTCCAG.

\section{Quantitation of DNA}

DNA concentration of PCR products was obtained through fluorimetric analysis of picogreen incorporation into dsDNA using the Picogreen dsDNA Quantitation Kit (Leiden, the Netherlands). Each PCR product was measured twice to avoid measurement artifacts. The PCR reactions from negative controls (without addition of DNA) were also measured to enable an estimation of the background caused by reagents and primer-dimers.

\section{RNA Extraction}

Total RNA was isolated using the GenElute Mammalian Total RNA Miniprep Kit (Sigma, Stockholm, Sweden) or AquaPure RNA Isolation Kit (Biorad, Hercules, California) according to the manufacturers' instructions. With both methods the final volume of the extracted RNA was $50 \mu \mathrm{l}$. One $\mu \mathrm{l}$ RNAsin (Promega UK, Southampton, United Kingdom) was immediately added, and the samples were stored at $-70^{\circ} \mathrm{C}$ until further processing.

\section{Microchip Gel Electrophoresis}

The analysis was performed using the Agilent 2100 Bioanalyser and an RNA 6000 Pico Labchip kit (Agilent Biotechnologies) according to the manufacturer's instructions. Total RNA was heated to $70^{\circ} \mathrm{C}$ for 2 minutes $1 \mu \mathrm{l}$ corresponding to $2 \%(\mathrm{v} / \mathrm{v})$ of each RNA preparation and then was transferred to the Pico Labchip, together with $1 \mu$ l of diluted RNA 6000 ladder (Ambion, Austin, Texas). Size distribution was determined from electropherograms. RNA amounts were calculated from the area under the curve by software provided by the manufacturer.

\section{Immunohistochemistry}

Antibodies and details regarding dilutions and choice of antigen retrieval are stated in Table 4. Paraffin sections of $4-\mu \mathrm{m}$ thickness were placed onto Superfrost plus slides (Mentzel), deparaffinized in xylen, and rehydrated in graded alcohols. For antigen retrieval, slides were immersed in $0.01 \mathrm{M}$ citrate buffer, $\mathrm{pH} 6.0$, and boiled for 7 minutes in a Decloacing chamber (Biocare Medical, Walnut Creek, California). Alternatively, slides were treated with $0.05 \%$ protease type VIII (Sigma, St. Louis, Missouri) for 10 minutes at room temperature. Endogenous peroxidase was blocked in $0.3 \% \mathrm{H}_{2} \mathrm{O}_{2}$ in PBS for 20 minutes. To reduce nonspecific binding of the primary antibody, sections were preincubated in $0.5 \%$ BSA-c (Aurion, Wageningen, the Netherlands) in PBS. Sections were incubated with primary antisera overnight at $4^{\circ} \mathrm{C}$. Incubation with antimouse peroxidase-conjugated Envision (DAKO, Glostrup, Denmark) was done at room temperature for 30 minutes. Diaminobenzidine (Sigma) was used as chromogen and Harris hematoxylin (Sigma) was used for counterstaining. Sections with known expression of the respective epitope were used as positive controls and parallel sections, whereas the primary antibody was replaced with PBS, serving as negative controls. Immunostained tissue array slides were evaluated and scored under the microscope.

\section{References}

Arnold MM, Srivastava S, Fredenburgh J, Stockard CR, Myers RB, and Grizzle WE (1996). Effects of fixation and tissue processing on immunohistochemical demonstration of specific antigens. Biotech Histochem 71:224-230.

Baker JR (1958). Principles of biological microtechnique. A study of fixation and dyeing, 1st ed. London: Methuen \& Co. Ltd.

Beckstead JH (1994). A simple technique for preservation of fixation-sensitive antigens in paraffin-embedded tissues. $\mathrm{J}$ Histochem Cytochem 42:1127-1134.

Brenes F, Harris S, Paz MO, Petrovic LM, and Scheuer PJ (1986). PLP fixation for combined routine histology and immunocytochemistry of liver biopsies. J Clin Pathol 39:459463. 
Crisan D and Mattson JC (1993). Retrospective DNA analysis using fixed tissue specimens. DNA Cell Biol 12:455-464.

Garnis-Jones S and Mah R (1988). Aceton fixation preserves melanoma-associated antigens in paraffin-embedded tissue. J Tu Marker Oncol 3:467-473.

Goldsworthy SM, Stockton PS, Trempus CS, Foley JF, and Maronpot RR (1999). Effects of fixation on RNA extraction and amplification from laser capture microdissected tissue. Mol Carcinog 25:86-91.

Gonzalez L, Anderson I, Deane D, Summers C, and Buxton D (2001). Detection of immune system cells in paraffin waxembedded ovine tissues. J Comp Pathol 125:41-47.

Greer CE, Lund JK, and Manos MM (1991). PCR amplification from paraffin-embedded tissues: Recommendations on fixatives for long-term storage and prospective studies. PCR Methods Appl 1:46-50.

Greer CE, Wheeler CM, and Manos MM (1994). Sample preparation and PCR amplification from paraffin-embedded tissues. PCR Methods Appl 3 (Suppl):113-122.

Herman GE, Chlipala E, Bochenski G, Sabin L, and Elfont E (1988). Zinc formalin fixative for automated tissue processing. J Histotechnol 11:85-89.

Huang SN, Minassian H, and More JD (1976). Application of immunofluorescent staining on paraffin sections improved by trypsin digestion. Lab Invest 35:383-390.

Johansson M, Jansson T, and Powell TL (2000). Na(+)-K(+)ATPase is distributed to microvillous and basal membrane of the syncytiotrophoblast in human placenta. Am J Physiol Regul Integr Comp Physiol 279:R287-294.

Lalani EN, Golding M, Hudson M, Chieffi G, Stamp G, Anilkumar TV, Sarraf C, and Alison MR (1995). Protein extraction and western blotting from methacarn-fixed tissue. J Pathol 177:323-328.

Ling G, Persson A, Berne B, Uhlen M, Lundeberg J, and Ponten F (2001). Persistent p53 mutations in single cells from normal human skin. Am J Pathol 159:1247-1253.

Mason JT and O'Leary TJ (1991). Effects of formaldehyde fixation on protein secondary structure: A calorimetric and infrared spectroscopic investigation. J Histochem Cytochem 39:225-229.

Nitta H, Munger W, Wilson E, Ralston R, and Alila H (1997). Improved in situ immunodetection of leukocytes on paraffinembedded mouse spleen. Cell Vis 4:73-80.
Persson AE, Ling G, Williams C, Backvall H, Ponten J, Ponten $F$, and Lundeberg $J(2000)$. Analysis of p53 mutations in single cells obtained from histological tissue sections. Anal Biochem 287:25-31.

Scheidl SJ, Nilsson S, Kalen M, Hellstrom M, Takemoto M, Hakansson J, and Lindahl P (2002). mRNA expression profiling of laser microbeam microdissected cells from slender embryonic structures. Am J Pathol 160:801-813.

Schutze K and Clement-Sengewald A (1994). Catch and move-cut or fuse. Nature 368:667-669.

Shi SR, Key ME, and Kalra KL (1991). Antigen retrieval in formalin-fixed, paraffin-embedded tissues: An enhancement method for immunohistochemical staining based on microwave oven heating of tissue sections. J Histochem Cytochem 39:741-748.

Shibutani M, Uneyama C, Miyazaki K, Toyoda K, and Hirose $M$ (2000). Methacarn fixation: A novel tool for analysis of gene expressions in paraffin-embedded tissue specimens. Lab Invest 80:199-208.

Tome Y, Hirohashi S, Noguchi M, and Shimosato Y (1990). Preservation of cluster 1 small cell lung cancer antigen in zinc-formalin fixative and its application to immunohistological diagnosis. Histopathology 16:469-474.

Wang BL, Springer GF, and Kaufman MW (1996). Concurrent immunohistochemical staining of tumor-infiltrating lymphocytes and carcinoma-associated $T$ (Thomsen-Friedenreich)/Tn antigens in human breast carcinoma. J Histochem Cytochem 44:187-191.

Warford A, Pringle JH, Hay J, Henderson SD, and Lauder I (1988). Southern blot analysis of DNA extracted from formalsaline fixed and paraffin wax embedded tissue. J Pathol 154:313-320.

Weaver DL, Mitchell J, and Leslie KO (1992). Effects of four primary fixatives on the sensitivity of immunohistochemical staining. J Histotechnol 15:27-30.

Williams C, Ponten F, Moberg C, Soderkvist P, Uhlen M, Ponten J, Sitbon G, and Lundeberg J (1999). A high frequency of sequence alterations is due to formalin fixation of archival specimens. Am J Pathol 155:1467-1471. 